OESOPHAGUS

\title{
Symptom association probability and symptom sensitivity index: preferable but still suboptimal predictors of response to high dose omeprazole
}

\section{S A Taghavi, M Ghasedi, M Saberi-Firoozi, M Alizadeh-Naeeni, K Bagheri-Lankarani, M J Kaviani, L Hamidpour}

Background: Twenty four hour oesophageal $\mathrm{pH}$ monitoring is frequently used to quantify the association between a patient's symptom and gastro-oesophageal reflux. Three indices (symptom association probability (SAP), symptom index (SI), and symptom sensitivity index (SSI)) are used to quantify and establish this relation.

Aims: The aim of the present study was to compare these indices against the omeprazole test (OT) as an objective indicator of reflux induced symptoms.

Methods: Fifty two patients with a predominant symptom of heartburn were enrolled from gastroenterology and primary care clinics. Baseline symptom score was calculated at the fist visit. All patients underwent 24 hour oesophageal $\mathrm{pH}$ monitoring, and symptom-reflux association indices were

See end of article for authors' affiliations

anch' afiliations

Correspondence to: Dr S A Taghavi, Department of Internal Medicine, Nemazee Hospital, Nemazee Square, Shiraz, Iran ataghavi@sums.ac.ir

Revised version received 23 March 2005

Accepted for publication 8 April 2005

Published online first

21 April 2005 calculated. All patients were placed on high dose omeprazole ( $40 \mathrm{mg}$ in the morning, $20 \mathrm{mg}$ at night) after completion of $\mathrm{pH}$ monitoring and symptom score was recorded again after one week.

Results: Thirty eight patients completed the study. All three indices were significantly related to each other $(p<0.001)$. SAP and SSI (with a new cut off of 1.3 , but not with the commonly used $5 \%$ ) had statistically significant relations with OT ( $p<0.05$ for both). SSI had the highest positive and negative predictive values and sensitivity. The specificity of SSI and SAP was equal and lower than SI. Areas under the receiver operating characteristic (ROC) curve for the three indices were not significantly different from each other or from a hypothetical non-discriminating test. Per cent time $\mathrm{pH}<4$, sex, and age had no relation to OT and no effect on its correlation with association indices. No cut off point could be found at which the results of SI could be related significantly to the OT results.

Conclusion: SAP and SSI are significantly related to symptomatic response to high dose omeprazole; however, they are far from perfect, with a significant number of discordant cases as well as relatively small areas under the ROC curves. We suggest that the new cut off of 1.3 be used for SSI in the future. Further research is needed to identify possible methods to improve the discrimination power of these indices or to identify possible patient characteristics that may affect this relation.
A mbulatory 24 hour $\mathrm{pH}$ monitoring is no longer universally accepted as "the gold standard"12 for diagnosis of gastro-oesophageal reflux (GOR) but it is routinely used for assessment of the possible associations between symptoms and reflux. ${ }^{2-4}$

Three measures (symptom sensitivity index (SSI), symptom index (SI), and symptom association probability (SAP)) were proposed as numerical scales to quantify the symptomreflux association, and therefore help to decide whether symptom episodes that occurred during the recording were related to GOR. ${ }^{2-6}$

SI is defined as the percentage of reflux related symptom episodes $^{6}$ and SSI the percentage of symptom related reflux episodes. ${ }^{3}$

These two indices however have common weak points: firstly, they have arbitrary cut off points, ${ }^{1}$ and secondly, they focus on the frequency of simultaneous occurrence of reflux and symptoms while ignoring the frequency of "nonassociated" reflux and symptom episodes. ${ }^{7}$

In order to circumvent these shortcomings, a new index (SAP), based on statistical analysis (cross tabulation) of a contingency table consisting of four possible combinations of reflux and symptoms, was suggested as a more statistically meaningful way of investigating this association ${ }^{3-8}$ and the $p$ value of $<0.05$ (Fisher's exact test) proposed as its cut off point. $^{247}$
Despite the apparent progress in sophistication, none of the above mentioned indices have been prospectively validated against an independent criterion of diagnostic accuracy, such as symptomatic response to antireflux therapy. ${ }^{2}$ In fact, the major problem is lack of an objective and reliable gold standard test-independent of $\mathrm{pH}$ monitoring-to which these indices can be compared. ${ }^{89}$

A number of studies suggest a short course of a high dose of a proton pump inhibitor (PPI) such as omeprazole as a practical, non-invasive, and relatively reliable test in establishing a cause and effect relation between GOR and symptoms. ${ }^{10-12}$ Although these "PPI tests" are far from ideal for diagnosing gastro-oesophageal reflux disease (GORD), ${ }^{8}$, they are the only available objective independent measures against which these indices can be tested. It also should be noted that the final purpose of an association index is to select those patients whose symptoms are related to acid reflux and therefore should be responsive to acid reduction therapy.

Abbreviations: GOR, gastro-oesophageal reflux; GORD, gastrooesophageal reflux disease; OT, omeprazole test; SAP, symptom association probability; SI, symptom index; SSI, symptom sensitivity index; ROC, receiver operating characteristic; PPI, proton pump inhibitor 
The aim of the present study was to prospectively validate SAP, SI, and SSI against symptomatic response to a PPI trial (omeprazole test $(\mathrm{OT})$ ).

\section{MATERIALS AND METHODS \\ Patients}

Fifty two consecutive patients with a predominant symptom of typical heartburn were initially enrolled in the study from gastroenterology and primary care clinics affiliated to Shiraz University of Medical Sciences. All patients consented to the protocol procedure.

Heartburn was defined as a burning sensation behind the sternum with radiation from the epigastrium towards the mouth. Inclusion was limited to patients with a history of at least three episodes of heartburn per week in the one month period prior to entry. Exclusion criteria were a history of peptic ulcer disease, gastrointestinal surgery, malignancy, use of PPIs in the previous four weeks, or $\mathrm{H}_{2}$ blockers and promotility agents in the previous week, and use of prescription non-steroidal anti-inflammatory drugs. Medication for other illnesses continued at constant doses when possible.

\section{Study protocol}

At entrance to the study a questionnaire, including demographic and upper endoscopy data and a heartburn severity and frequency score, ${ }^{10-12}$ was completed. Total heartburn score was calculated by multiplying grades of severity and frequency (table 1).

Endoscopy data were not used in the final analysis because the reliability of the initial data could not be confirmed, and $60 \%$ of patients did not consent to repeat endoscopy. Later, all patients underwent ambulatory oesophageal $\mathrm{pH}$ monitoring and then a trial of high dose omeprazole (generic omeprazole; Exir Pharmaceutical Company, formerly Lorestan Pharmaceutical Company, Lorestan, Iran, copied from UQUIFA Pharmaceutical Company Spain), $40 \mathrm{mg}$ in the morning, $20 \mathrm{mg}$ at night, for seven days.

The same questionnaire was completed again at the end of the omeprazole trial by one of the investigators, unaware of the $\mathrm{pH}$ monitoring results. A reduction of $>50 \%$ in the heartburn score or reaching zero from any previous score was considered a positive OT. ${ }^{11}$

\section{Ambulatory 24 hour pH monitoring}

After an overnight fast, the pH probe (Zinetics $24 \mathrm{ME}$; Medtronic Functional Diagnostics, Tonsbakken, Denmark) was positioned transnasally $5 \mathrm{~cm}$ above the manometrically determined lower oesophageal sphincter and secured to the nose and cheek. A reference electrode was attached to the chest skin. The $\mathrm{pH}$ electrode was calibrated before and after the recording using $\mathrm{pH} 7$ and 1 buffer solutions.

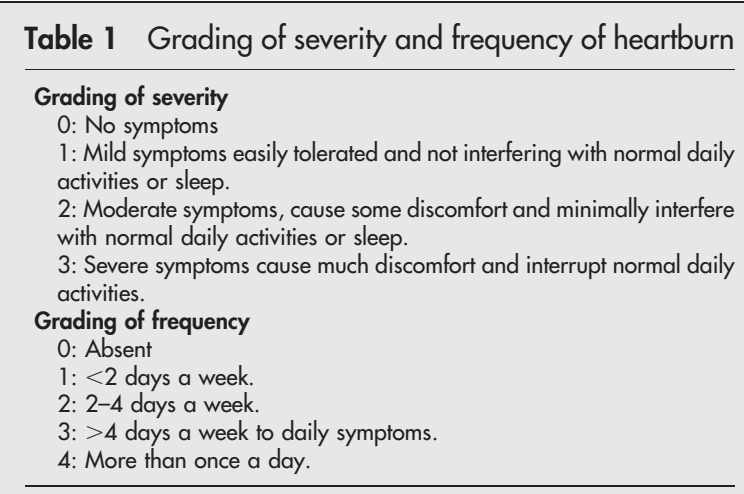

\begin{tabular}{|c|c|}
\hline $\begin{array}{l}\text { Age (y) (mean (range)) } \\
\text { Sex (M/F) } \\
\text { Smoker/non-smoker } \\
\text { Alcoholic/non-alcoholic }\end{array}$ & $\begin{array}{l}37.45(23-64) \\
20 / 18 \\
4 / 31^{*} \\
2 / 33 \dagger\end{array}$ \\
\hline
\end{tabular}

\begin{tabular}{|c|c|c|}
\hline & $\begin{array}{l}\text { Symptom } \\
\text { episodes }\end{array}$ & $\begin{array}{l}\text { Reflux } \\
\text { episodes }\end{array}$ \\
\hline Min & 1 & 5 \\
\hline Max & 18 & 768 \\
\hline Mean & 3.71 & 120.76 \\
\hline SD & 3.7 & 171.6 \\
\hline Sum & 141 & 4589 \\
\hline
\end{tabular}

Patients registered periods of eating, supine position, and heartburn using a written diary as well as pressing buttons on the data logger. Medtronic $\mu$ digitrapper $4 \mathrm{Mb}$ flash series (Synetics Medical AB, Stockholm, Sweden) was used as the data logger. Patients were encouraged to pursue their usual everyday activities and diet. After completion of the study, recorded data were transferred to a computer and analysed using dedicated software (Multigram, GI edition, version 6.31, Gastrosoft Inc). All tracings were reviewed by one of the investigators to reveal any technical difficulties.

\section{Data analysis}

SI and SSI were calculated as described by Wiener and colleagues ${ }^{6}$ and Breumelhof and colleagues, ${ }^{3}$ respectively. For calculation of SAP, consecutive two minute periods of $\mathrm{pH}$ recording were analysed for the presence of reflux and symptoms. A symptom episode was considered as reflux positive if either of the two minute periods preceding or following the start of the symptom was reflux positive $(\mathrm{pH}$ $<4$ for $\geqslant 5$ seconds). A contingency table was constructed and Fisher's exact test was used to calculate the $p$ value according to Weusten and colleagues. ${ }^{4}$ SAP was calculated as $(1.0-\mathrm{P}) \times 100 \%$.

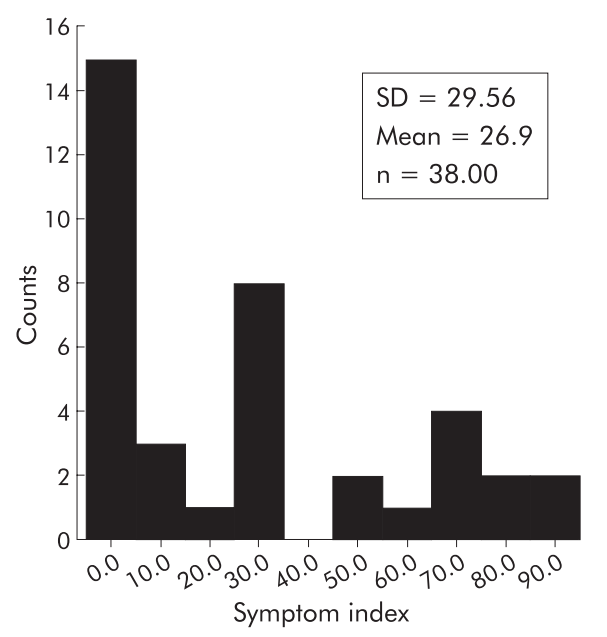

Figure 1 Distribution of symptom index in the study population. 


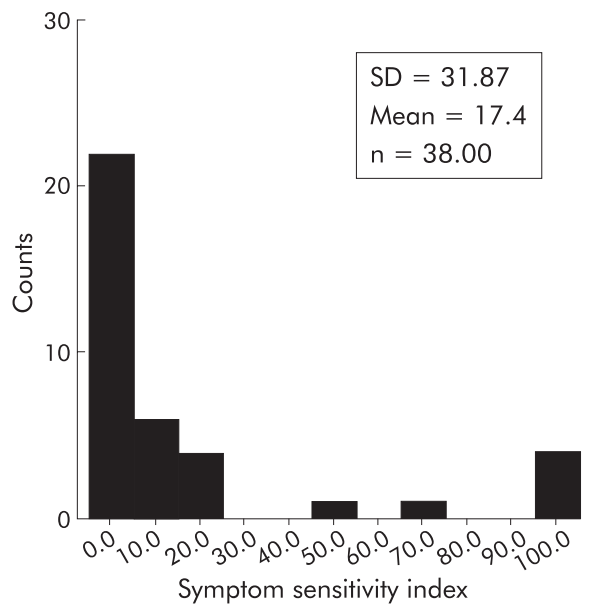

Figure 2 Distribution of symptom sensitivity index in the study population.

Values $>1.3$ (based on the findings of the current study), $\geqslant 50 \%$, and $>95 \%$ were considered as indicators of significant symptom-reflux association for SSI, SI, and SAP, respectively. ${ }^{4}$ Cross tabulation and the $\chi^{2}$ test were used to identify any significant relationships between the results of the OT (responder, non-responder) and each association parameter (reflux associated, non-reflux associated).

Statistical analyses were done by SPSS statistical software package(version 11.5.0 SPSS Inc, 1989-2002). Comparison of receiver operating characteristic (ROC) curves was performed using MedCalc statistical software package (version 7.6.0.0, 1993-2004).

\section{RESULTS}

Fifty two patients were initially enrolled into the study. Fourteen had to be withdrawn from further analysis for various protocol violations: seven due to lack of symptoms during the $\mathrm{pH}$ recording period, six due to unacceptable quality of recorded data (significant error in post test calibration of $\mathrm{pH}$ electrode in three and electrical interferences, presumably due to loosening of the external reference electrode, in three), and one patient could not tolerate the catheter for more than six hours. Patient's characteristics are summarised in table 2 .

A total number of 4589 reflux episodes and 141 symptom episodes were registered in the 38 patients who completed the study. Table 3 shows the statistical characteristics of the registered reflux and symptom episodes.

Mean acid exposure time $(\mathrm{pH}<4)$ in the studied groups was 128.1 minutes (4-1444). Mean per cent time during ambulatory $\mathrm{pH}$ monitoring in which $\mathrm{pH}$ was $<4$ was $9.1 \%$ (SD 2.37; range $0.1-53.7 \%$ ): $42.1 \%$ of patients had $\mathrm{pH}<4$ for more than $4 \%$ of the recoded time. Figures $1-3$ show the distribution of SI, SSI, and SAP in these patients.

The OT was positive in 23 patients $(51.1 \%)$. In the OT positive group, $13(56.52 \%)$ had complete disappearance of symptoms and $10(43.48 \%)$ had more than 50\% improvement in symptom score. Of those patients with a negative test, nine $(60 \%)$ had less than 50\% improvement and six $(40 \%)$ had no change in the symptom score. No age or sex differences were demonstrated between omeprazole responders and nonresponders ( $\mathrm{p}$ values 0.611 and 0.214 , respectively). There were also no significant differences between OT positive versus negative groups in total time $\mathrm{pH}<4 \quad(\mathrm{p}=0.497$, Komolgorov-Smirnov test).

Although there were more patients with abnormal pH monitoring results in the omeprazole responder versus

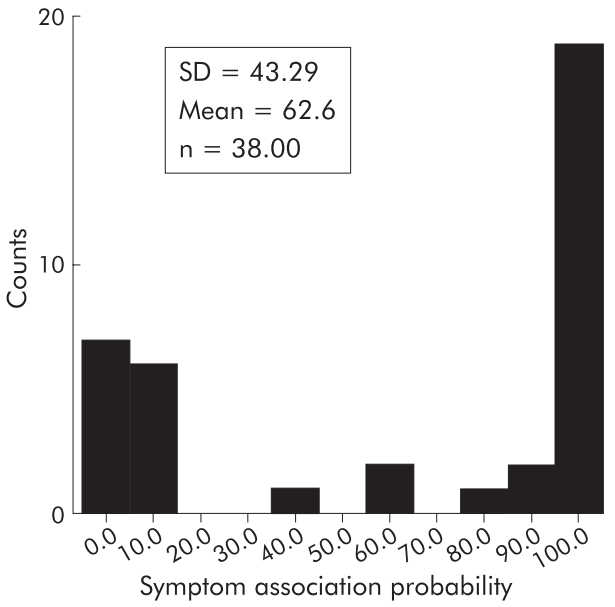

Figure 3 Distribution of symptom association probability in the study population.

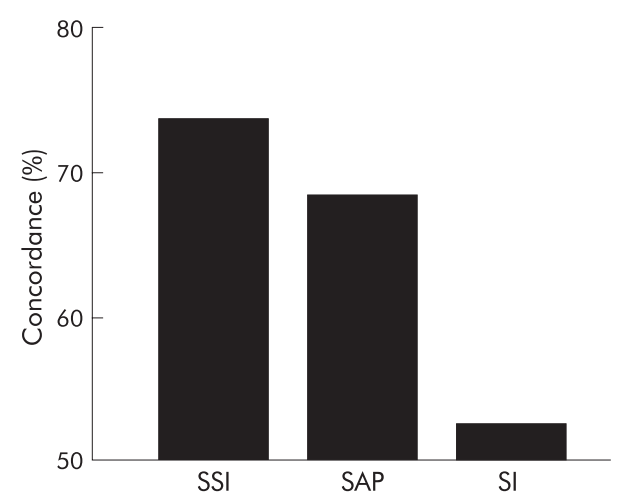

Figure 4 Per cent concordance of symptom sensitivity index (SSI) symptom association probability (SAP), and symptom index (SI) with the omeprazole test.

Table 4 Comparison of predictive values, sensitivity, and specificities for the symptom association probability (SAP), symptom sensitivity index (SSI), and symptom index (SI)

\begin{tabular}{lllll}
\hline & $\begin{array}{l}\text { Positive } \\
\text { predictive value }\end{array}$ & $\begin{array}{l}\text { Negative } \\
\text { predictive value }\end{array}$ & Specificity & Sensitivity \\
\hline SAP & 78.95 & 57.89 & 73.33 & 65.22 \\
SSI & 80.95 & 64.71 & 73.33 & 73.91 \\
SI & 72.73 & 44.44 & 80 & 34.78 \\
\hline
\end{tabular}

non-responder group (52.2\% $v 36.3 \%)$, the difference was not statistically significant (Pearson $\chi^{2}=2.423, \mathrm{p}=0.182$ ).

A statistically significant association was found between the OT results and SAP (Pearson $\chi^{2}=5.397$, two sided significance $=0.02$, Fisher's exact test 0.045 and 0.022 for two and one sided significance, respectively). No significant relation was found between OT and SSI (using cut offs of 5\% and $10 \%$ ) (Pearson $\chi^{2} 2.42$ and 1.54 and $p$ values 0.12 and 0.22 , respectively). However, the relation was significant when a level of more than 1.3 (the most suitable point on the ROC curve) was used as the cut off (Pearson $\chi^{2}=8.19$, $\mathrm{p}<0.05)$. There was no significant relation between SI and the results of the OT using significance levels of 10, 15, 20, 25, 30, $35,40,45,50,55,60,65,70,75,80,85,90$, and 95 ( $\mathrm{p}$ value 
Table 5 Comparison of mean number of symptom episodes in the three groups classified on the basis of correlations between the omeprazole test (OT) and symptom association probability (SAP) or symptom sensitivity index (SSI)

\begin{tabular}{llll}
\hline & $\begin{array}{l}\text { Omeprazole test } \\
\text { positive/index negative }\end{array}$ & $\begin{array}{l}\text { Concordant (both OT } \\
\text { and index negative or } \\
\text { positive) }\end{array}$ & $\begin{array}{l}\text { Omeprazole test } \\
\text { negative/index positive }\end{array}$ \\
\hline SAP & $1.63(1-2.25)$ & $4.27(2.56-5.98)$ & $4.25(3.45-5.05)$ \\
SSI & $1.33(0.79-1.88)$ & $4.14(2.55-5.73)$ & $4.25(3.45-5.05)$ \\
\hline \multicolumn{2}{l}{ Values are mean (95\% confidence interval). }
\end{tabular}

$>0.05$, Pearson $\chi^{2}$ ). Figure 4 shows the concordance rate of each of the association indices with OT.

Although SSI had the highest concordance rate with OT, it was unable to predict its results in $26.3 \%$ of patients. There were no differences in sex, age, or level of acid exposure between the concordant versus discordant groups for either SAP or SSI. SSI also had the highest sensitivity and negative predictive value for OT (table 4). It should be noted that with the commonly used level of $>5 \%$, SSI had lower concordance, sensitivity, and predictive values than SAP. The specificity of SSI and SAP was equal and less than SI (table 4).

Statistically significant associations were found between SAP and the two other variables (SI and SSI) using bivariate correlation (Spearman's rho 0.793 and 0.604 and p value 0.000 for both). SI and SSI were also significantly related ( Spearman's rho $=0.677$ and $p$ value $=0.000$ ). Mean SSI was significantly higher in the OT positive versus the OT negative group (Komolgorov-Smirnov test $\mathrm{Z}=1.424, \mathrm{p}=0.035$ ) but the difference for the two other indices was not statistically significant.

Comparison of mean numbers of symptom episodes in the group with a positive OT and negative SAP with both concordant groups and the group with a negative OT and a positive SAP showed significantly lower numbers of symptom episodes in the first group (Kruskall-Wallis nonparametric test, $\mathrm{p}<0.05$ for both relations). The same was also true for the mean number of symptom episodes and SSI (Kruskall-Wallis non-parametric test, $\mathrm{p}<0.05$ for both relations) (table 5). No difference was found for the number of reflux episodes between these groups.

Construction of ROC (receiver operating characteristic) curves for the three association indices showed that although SSI had the largest area under the curve, none of the indices had enough discrimination power to be significantly different from a hypothetical non-discriminating test in prediction of the results of OT (fig 5). There were no statistically significant differences between area under the ROC curve for each of the above mentioned indices ( $\mathrm{p}>0.05$ for all, Hanley and McNeil method).

\section{DISCUSSION}

In our study, SI had the poorest performance of all association indices, with the highest discordance rate as well as absence of any cut off point at which the relation of its results with OT could reach a statistically significant level. Considering the fact that the final purpose of an association index is to provide a yes or no answer to the question of reflux-symptom association, absence of an objective meaningful cut off point makes SI almost useless.

SAP and SSI (with the new cut off point) had statistically significant associations with the results of the OT but their associations were far from ideal, with discordance rates of $32 \%$ and $26 \%$, respectively. SSI had the highest positive and negative predictive values, sensitivity, and concordance for the results of OT.

As SAP is a statistical parameter showing the presence or absence of a non-random relation between acid exposure (measured on the basis of the selected threshold) and perceived symptoms, the presence of discordance between SAP and OT reveals major defects in our understanding of the symptom production process in GORD. The superior performance of SSI in terms of sensitivity, concordance, and predictive values compared with SAP, despite the more statistically sound basis for calculation of the latter, also cannot be explained easily and may be related to the same defects. Theses defects may include inability of the present indices to take into account the possible role of non-acid reflux and application of an identical threshold in different patients with different symptoms and (most likely) different levels of visceral sensitivity. It should be noted that the imperfect nature of OT as a gold standard may also affect these relations. Also, the areas under the ROC curves for these indices were not significantly different from each other and therefore the possible practical importance of the modest differences observed between their sensitivities, specificities, and predictive values will be minimal.

With all three indices, the number of discordant cases with a positive OT and a negative association index was higher than discordant cases with the reverse situation
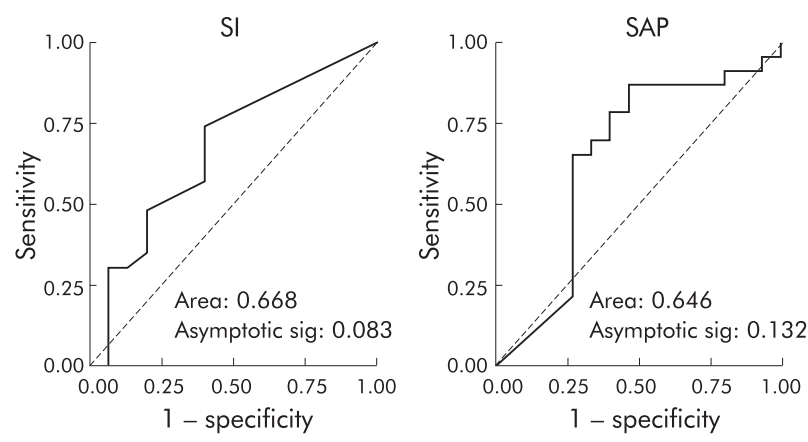

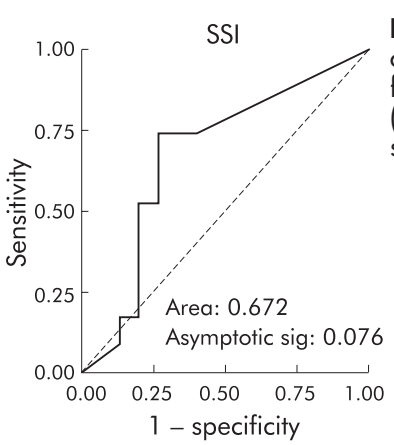

Figure 5 Comparison of receiver operating characteristic (ROC) curves for symptom association probability (SAP), symptom index (SI), and symptom sensitivity index (SSI). 
( $21 \% \vee 10.5 \%$ for SAP). For $21 \%$ with a positive OT but a negative SAP, placebo response to omeprazole with no real reflux induced symptom is one of the possibilities which can be tested in a double blind placebo controlled study. On the other hand, this group had a significantly lower number of symptom episodes compared with the two other groups (concordant group and those with a negative OT and positive SAP). It is noteworthy that the same relation was found between SSI and number of symptom episodes (table 5). A possible explanation is that these association indices may have lower sensitivities for detection of symptom-reflux associations in patients with lower numbers of symptoms during $\mathrm{pH}$ recordings and may require modifications in this group.

For the group with a negative OT and positive SAP, repeat $\mathrm{pH}$ monitoring while receiving omeprazole may show whether this combination is due to the ineffectiveness of omeprazole in suppressing acid reflux, or whether symptoms are induced by factors other than acid reflux. The relatively low percentage of patients with significant acid reflux in our study population-all suffering from symptoms that are considered typical of GOR-was also reported in other recent articles $^{13}$ and may be related to differences in perception of constant stimuli between different patients. ${ }^{14}$

Contrary to previous studies ${ }^{4}$ in which SAP had a significant relation only with SI, in our study, all of these indices were significantly related to each other. Failure of the association indices to predict the results of OT better than a hypothetical non-discriminating test, as shown by the ROC curves, may be related to their inherent shortcomingswhich may not be able to account for all of the factors involved in symptom production in GOR-as well as the inability of the OT to serve as the gold standard for discrimination of reflux related symptoms. However, it is apparent that these indices are unable to predict the response of the one of the most typical symptoms of GOR (heartburn) to one of the most effective medical treatments available.

It should be noted that the precision of the estimates of sensitivity, specificity, significance of the relations, and results of ROC curves are dependent on the number of subjects studied and future studies with more patients are needed to document the results of the present investigation.

\section{ACKNOWLEDGEMENTS}

We are indebted to the staff of the Nemazee Hospital Endoscopy Unit. This study was supported by a grant from the research council of the Shiraz University of Medical Sciences.

\section{Authors' affiliations \\ S A Taghavi, M Saberi-Firoozi, M Alizadeh-Naeeni, K Bagheri- Lankarani, M J Kaviani, L Hamidpour, Gastroenterohepatology Research Centre, Shiraz University of Medical Sciences, Shiraz, Iran M Ghasedi, Arak University of Medical Sciences, Arak, Iran \\ Conflict of interest: None declared.}

\section{REFERENCES}

1 Bate CM, Riley SA, Chapman RWG, et al. Evaluation of omeprazole as a costeffective diagnostic test for gastro-oesophageal reflux disease. Aliment Pharmacol Ther 1999; 13:59-66.

2 American Gastroenterological Association Medical Position Statement: Guidelines on the use of esophageal pH-recording. Gastroenterology 1996; 110:1981-96.

3 Breumelhof $R$, Smout AJPM. The symptom sensitivity index: A valuable additional parameter in 24-hour esophageal pH recording. Am J Gastroenterol 1991;86:160-4.

4 Weusten BL, Rolfs JMM, Akkermans LMA, et al. The symptom association probability: An improved method for symptom analysis of 24-hour esophageal pH data. Gastroenterology 1994;107:1741-5.

5 Shi G, Bruley D, Varannes S, et al. Reflux related symptoms in patients with normal oesophageal exposure to acid. Gut 1995;37:457-64.

6 Wiener GJ, Richter JE, Copper JB, et al. The symptom index: A clinically important parameter of ambulatory 24 -hour esophageal $\mathrm{pH}$ monitoring. Am J Gastroenterol 1988;83:358-61.

7 Orr WC. The physiology and philosophy of cause and effect. Gastroenterology 1994;107:1897-901.

8 Numans ME, Lau J, de Wit NJ, et al. Bonis short-term treatment with protonpump inhibitors as a test for gastroesophageal reflux disease: a meta-analysis of diagnostic test characteristics. Ann Intern Med 2004;140:518-27.

9 Numans ME, Bonis PA, Lau J. Limitations of gold standards for diagnosing gastroesophageal reflux disease. Ann Intern Med 2004;141:648-9.

10 Fass R, Fennerty MB, Ofman JJ, et al. The clinical and economic value of a short course of omeprazole in patients with noncardiac chest pain. Gastroenterology 1998;115:42-9.

11 Fass R, Ofman JJ, Sampliner RE, et al. The omeprazole test is as sensitive as $24-\mathrm{h}$ oesophageal $\mathrm{pH}$ monitoring in diagnosing gastro-oesophageal reflux disease in symptomatic patients with erosive esophagitis. Almient Pharamcol Ther 2000; 14:389-96.

12 Schenk BE, Kuipers EJ, Klinkenberg-Knol EC, et al. Omeprazole as a diagnostic tool in gastroesophageal reflux disease. Am J Gastroenterol 1997;92: 1997-2000

13 Colas-Atger E, Bonaz B, Papillon E, et al. Relationship between acid reflux episodes and gastro-esophageal reflux symptoms is very inconstant. Dig Dis Sci 2002:47:645-51.

14 Crawford Clark W. Pain sensitivity and the report of pain: An introduction to sensory decision theory. Anesthesiology 1974;3:272-87.

\section{EDITOR'S QUIZ: GI SNAPSHOT}

\begin{abstract}
Answer
From question on page 1061

Her symptoms gradually improved with discontinuation of indomethacin. Follow up colonoscopy showed that the diaphragm-like stricture remained with circumferential scar formation. A diagnosis of non-steroidal anti-inflammatory drug (NSAID) induced colonic ulcer and stricture was made on the basis of her clinical course, as well as a characteristic gross and microscopic appearance. During one year of follow up, haematochezia has not recurred and the stricture has been free from obstruction with stool softener. A rare but increasingly recognised disorder known as diaphragm disease has become pathognostic for NSAID induced colonic ulcer and stricture. The lesions usually involve the proximal colon and are associated with sustained release formulations of NSAIDs. Local and/or systemic effects of NSAIDs damage the intestinal mucosa, presumably by inhibition of cyclooxygenase and increase in permeability. Cessation of NSAIDs is an essential treatment. Management with sulphasalazine and metronidazole has been reported. Endoscopic balloon dilatation or segmental resection are indicated for the stricture when medical treatments fail. In conclusion, colonic diaphragm disease should be considered in the management of abdominal symptoms of patients on NSAIDs.
\end{abstract}

